\title{
Chromosome Structure
}

National Cancer Institute

\section{Source}

National Cancer Institute. Chromosome Structure. NCI Thesaurus. Code C13377.

The 3-D org anization of histones and other proteins on chromosomal DNA in the nucleus and allow for its form and function during cellular growth and division. 